\title{
Erroneous Results from SPICE Simulations of Switching Converters: A Dynamical System Viewpoint
}

\author{
Xiaoqun $\mathrm{Wu}^{* \dagger}$, Siu-Chung Wong*, Chi K. Tse* and Junan $\mathrm{Lu}^{\dagger}$ \\ *Department of Electronic and Information Engineering, Hong Kong Polytechnic University, Hong Kong, China \\ ${ }^{\dagger}$ School of Mathematics and Statistics, Wuhan University, Hubei 430072, China
}

\begin{abstract}
The SPICE simulation program is widely used as a brute force simulator for analyzing and designing switching power converters. Results from SPICE are mostly useful, but their integrity is sometimes questionable as erroneous results could be obtained which may not reflect the true behavior of the circuits being simulated. Various parameters in SPICE are crucial in controlling the convergence and accuracy of the simulated results, e.g., relative error tolerance and maximum integration step size. In this paper, we study the system consisting of the SPICE simulation algorithm and the circuit being simulated. Specifically, we describe the generation of flawed solutions in terms of bifurcation of the system under parameter variations. Erroneous results have been collected for different relative error tolerances, maximum integration step sizes, and parasitic inductance and capacitance. These flawed solutions can be analyzed in terms of the manifestation of period-doubling bifurcation and chaotic behavior under variation of selected simulation parameters. This paper provides a systematic approach to rationalizing the behavior of the SPICE simulator, its practical significance being in the identification of the ranges of simulation parameters for which flawed solutions can be produced.
\end{abstract}

\section{INTRODUCTION}

Proper models and reliable simulations are indispensable to the analysis and design of power electronics circuits [1]. SPICE is among the most widely used simulation tools which provide design information such as circuit operation, stability, transient performance, device stresses, etc [1]-[5]. Transient analysis using SPICE is the most popular method for studying the large-signal behavior of switching converters. Switching converters, being nonlinear and time-varying, can be difficult to simulate using SPICE [6]-[8], the most commonly encountered problem being convergence. To overcome the convergence problems, engineers often resort to trial-and-error types of adjustment of simulation parameters, such as relaxing the relative error tolerance and increasing the maximum step size. Furthermore, flawed or erroneous results may be generated, giving wrong or misleading design information [9]. It is thus of interest to study the way in which the SPICE simulator fails to give correct results. So far, very little work has been reported to address the dynamics of the simulation process from a system viewpoint (i.e., treating the simulator that imitates the dynamics of the switching converter as a dynamical system and its parameters as system/bifurcation parameters), and to study the effects on the integrity of the results obtained. In this paper we investigate the SPICE simulator and its

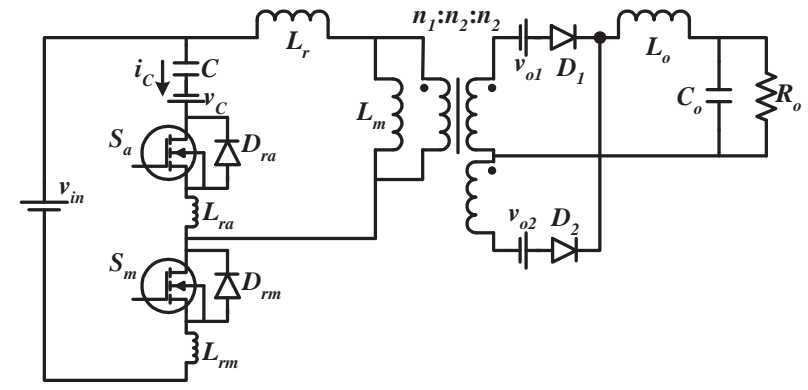

Fig. 1. Asymmetrical half-bridge forward converter.

dynamical behavior. We wish to know what parameters control the integrity of the results and how flawed results normally develop as system parameters are changed. The purpose is to understand the dynamics of SPICE simulations, leading to a more informed use of SPICE in the design of switching converters.

In Section II, we first describe a switching converter and some typical simulation results from SPICE. We will give 3 sets of simulation results in Section II-B. These results show different behaviors of the same circuit, under the same circuit condition but with different simulator's parameters. We treat the SPICE algorithm and the switching converter as a nonlinear dynamical system [10]-[12] and study the effects of the simulator's parameters, RELTOL (relative error tolerance), TMAX (maximum step size), parasitic inductors $L_{r a}, L_{r m}$, and parasitic capacitance $C_{j o}$, on the simulator's outputs. One of the circuit variables is chosen as the output. The simulated outputs of the system for different values of RELTOL are reported in Section III-A, those for different values of TMAX are reported in Section III-B, and those for varying parasitic inductance and capacitance are reported in Section III-C. We illustrate in Section IV the different operation boundaries in the system parameter space within which correct or flawed results are produced. Finally, we conclude our findings in Section V.

\section{SPICE Simulation OF SWitching CONVERTERS}

We consider the half-bridge forward converter shown in Fig. 1 [13], [14]. SPICE has been found very useful in identifying the number of states of operation, zero-voltage 


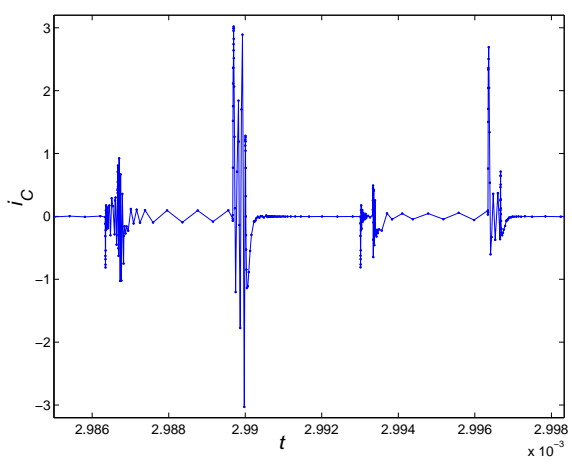

(a)

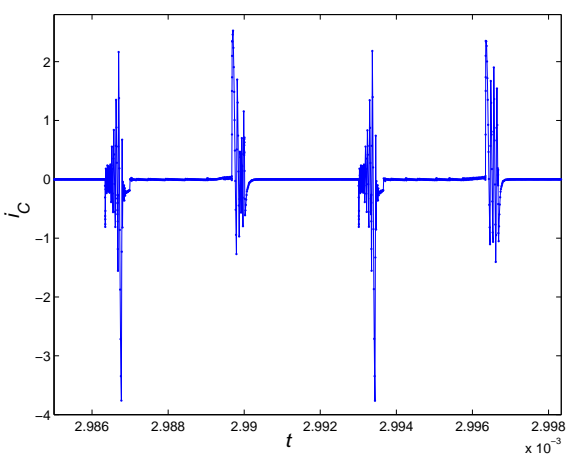

(b)

Fig. 2. Flawed simulations of $i_{C}$ with (a) RELTOL $=0.005$ and default TMAX; (b) RELTOL $=0.02$ and TMAX $=10 \mathrm{~ns}$.

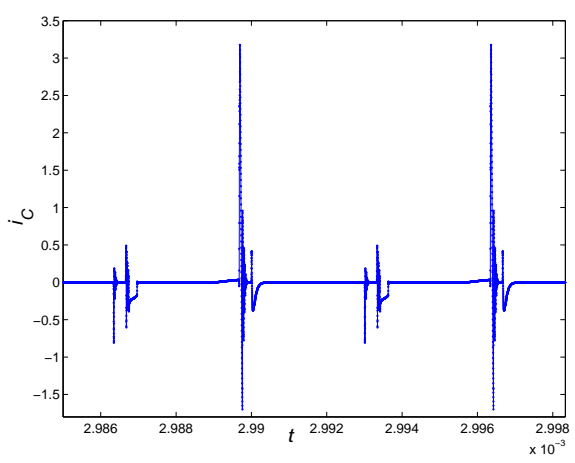

Fig. 3. Improved simulations of $i_{C}$ with RELTOL $=0.002$ and TMAX $=1$ ns

switching conditions, device stresses and sensitivity of this circuit. In the following study, we will use SPICE to verify the operational waveforms of the circuit and our purpose is to find out whether SPICE would faithfully reproduce the correct information and under what conditions it would fail to do so. Furthermore, as we are interested in knowing the integrity of SPICE in simulating the correct waveforms, we are not concerned here with the problem of convergence associated with the presence of a feedback loop. We thus omit such a feedback loop and focus on openloop simulations which are often performed by engineers in order to inspect the operational waveforms.
TABLE I

SIMULATION TIME FOR DIFFERENT RELTOL AND TMAX

\begin{tabular}{ccc}
\hline RELTOL & TMAX & Simulation time \\
\hline 0.005 & default & $5.780 \mathrm{~s}$ \\
0.02 & $10 \mathrm{~ns}$ & $17.766 \mathrm{~s}$ \\
0.002 & $1 \mathrm{~ns}$ & $151.904 \mathrm{~s}$ \\
\hline
\end{tabular}

\section{A. Circuit Description}

The circuit in Fig. 1 can be described in a SPICE netlist file (omitted here). Dummy sources $v_{C}, V_{o 1}$ and $V_{o 2}$ are added for the measurement of current. The transformer is assumed ideal. Parasitic source-pin-inductors $L_{r a}$ and $L_{r m}$ of the MOS switches are added for more accurate waveform calculations. $D_{r a}$ and $D_{r m}$ are body diodes of the MOS transistors. The two diodes can also be shunted by external fast switching diodes to improve the efficiency.

Steady-state (or near steady-state) waveforms can be obtained by running transient analysis in SPICE for a sufficiently long period of time. Usually, steady-state waveforms can be assumed when there is no significant change of the waveforms in two consecutive switching periods. A typical transient analysis command is as follows:

. TRAN TSTEP TSTOP < TSTART < TMAX >>

where TMAX is the maximum step size used by SPICE. As a default, the program chooses either TSTEP or (TSTOPTSTART)/50.0, whichever is smaller. The calculation of steady-state waveforms can be speeded up by using the optional UIC (use initial conditions) keyword at the end of the .TRAN statement. Also, near steady-state voltages and currents values are inserted using "IC=..." at the end of the elements or using the .IC control line for various initial node voltages obtained from previous solutions.

\section{B. Erroneous Simulation Results: Beyond Speed-Accuracy Tradeoff}

It is generally believed that simulation speed and convergence can be improved dramatically by relaxing the requirement of simulation tolerance [6]. We have measured the simulation time for three sets of simulation tolerances and controls, as shown in Table I. Figs. 2 and 3 show the corresponding simulated waveforms.

For comparison, we also present in Fig. 4 the "correct" (practically consistent) waveforms of $i_{C}$ with a tighter simulation control, i.e., RELTOL $=0.001$, TMAX $=1 \mathrm{~ns}$ and the integration method is Gear. We observe a rather large discrepancy in the simulated results for different simulator's parameters. This clearly shows that the simulator's parameters do not only control the speed-accuracy tradeoff, but may also be crucial in determining whether correct or flawed results would be produced. It is thus useful to study the dynamics of the system consisting of the SPICE simulation algorithm that imitates the dynamics of the switching converter.

Since we are primarily concerned with system stability in relation to the SPICE parameters (RELTOL and TMAX), and the circuit parameters (parasitic inductors $L_{r a}, L_{r m}$ and 


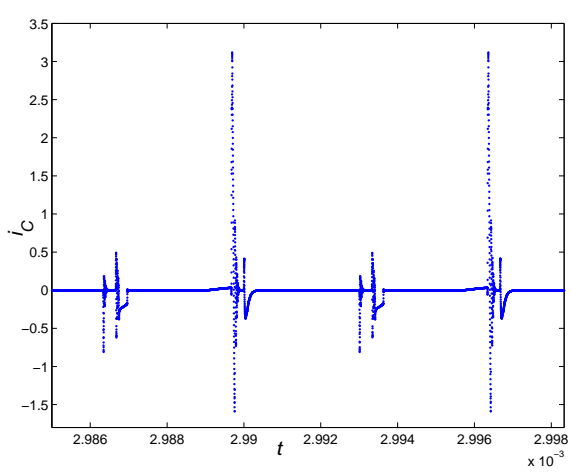

(a)

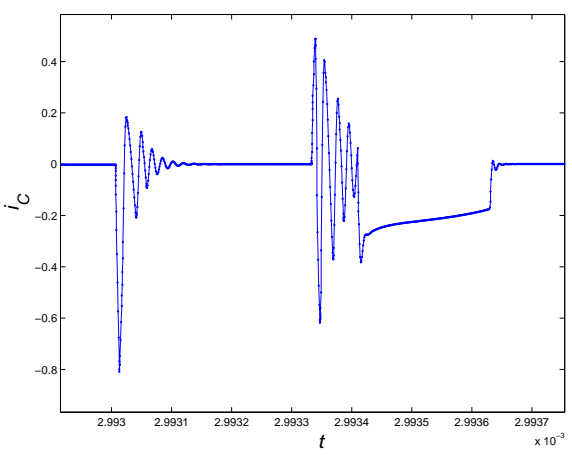

(b)

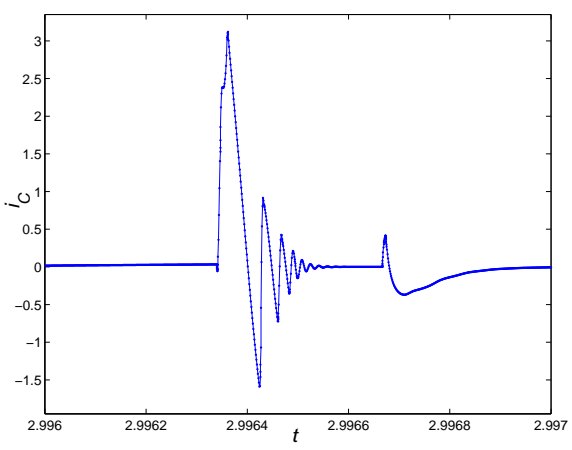

(c)

Fig. 4. (a) "Correct" simulation of $i_{C}$ with RELTOL $=0.001$, TMAX $=1$ ns and METHOD = Gear; (b)-(c) close-up views.

capacitance $C_{j o}$ of body diodes $D_{r a}$ and $D_{r m}$ ), we will focus on variation of these parameters. From the SPICE simulation results, we observe period-doubling bifurcation and chaotic behavior in some intervals of time during a switching cycle. In the following, we choose the current $i_{C}$, as shown in Fig. 1, as the system output for the purpose of identifying the instability phenomena.

\section{Period-Doubling Bifurcation of SPICE Results}

\section{A. Varying RELTOL}

In this section, we present a detailed inspection of the effect of varying RELTOL. In these simulations, TMAX is fixed at $5 \mathrm{~ns}$. The simulated results with varying RELTOL are shown in Figs. 5 to 8.

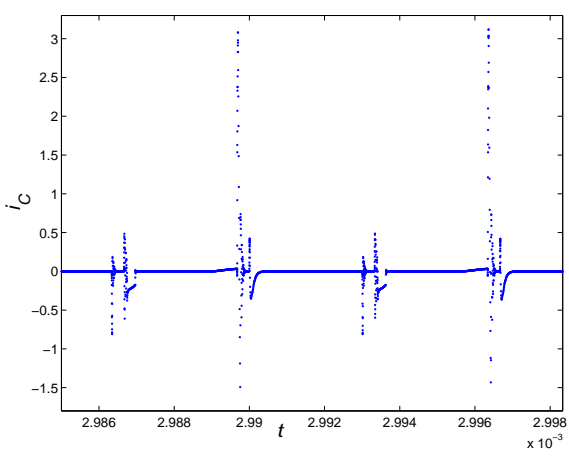

(a)

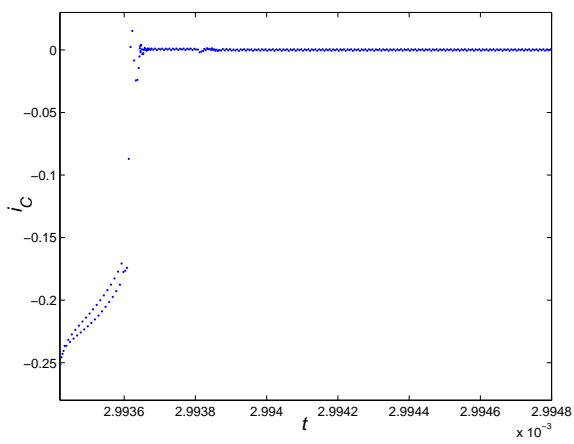

(b)

Fig. 5. (a) Flawed periodic operation of $i_{C}$ with RELTOL $=0.001$ and (b) close-up view.

Fig. 5 plots the waveform of $i_{C}$ in two switching cycles with RELTOL $=0.001$. An erroneous period-2 subharmonic operation is observed, as shown in the close-up view in Fig. 5(b). Fig. 6 shows period- 2 and period- 4 operation (also erroneous) in some intervals of a switching cycle with REL$\mathrm{TOL}=0.01$. Also, Fig. 7 shows erroneous periodic operation of longer periods with a larger RELTOL. Finally, Fig. 8 displays erroneous chaotic operation with RELTOL $=0.2$.

In general, we observe that the simulation results become more "unstable" (in the sense that more erroneous answers are produced) as we increase RELTOL.

\section{B. Varying TMAX}

We now look at the effect of varying TMAX. For simplicity, we keep RELTOL at 0.01 , and display the close-up views in some interval of a switching cycle.

Fig. 9 (a) displays the "correct" simulated operation of $i_{C}$ with TMAX $=0.5 \mathrm{~ns}$. When TMAX is relaxed to $2 \mathrm{~ns}$, an erroneous period- 2 operation is observed, as shown in Fig. 9 (b). When we further increase TMAX, we observe period-2 and period-4 operations, as shown in Figs. 9 (c) to (d).

Thus, we observe that the simulation results again become progressively more "unstable" as TMAX is increased. Again, by "unstable" we mean that the simulation results do not converge to the correct results.

\section{Varying parasitic inductance and capacitance}

Since parasitic inductors $L_{r a}, L_{r m}$ and parasitic capacitance $C_{j o}$ of the body diodes of the MOS transistors may also affect 


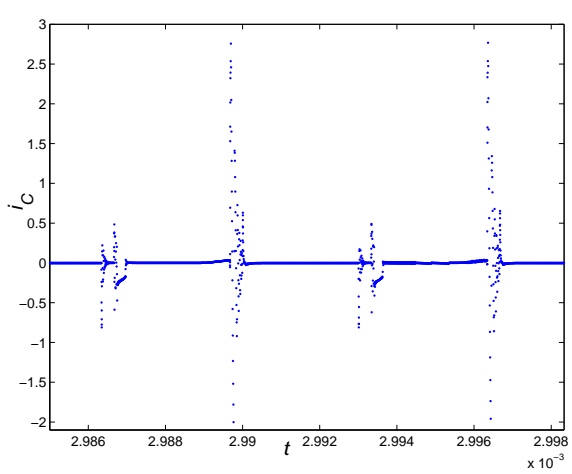

(a)

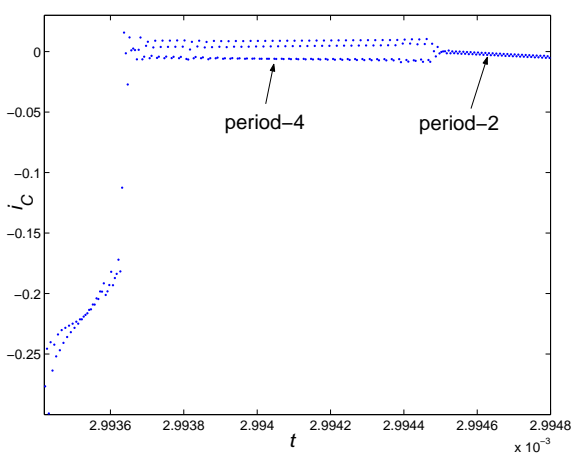

(b)

Fig. 6. (a) Flawed periodic operation of $i_{C}$ with RELTOL $=0.01$ and (b) close-up view.

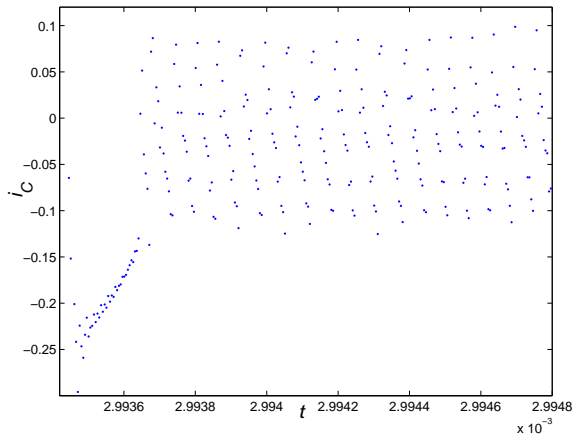

Fig. 7. Close-up view of flawed periodic operation of $i_{C}$ of longer period with $\mathrm{RELTOL}=0.1$

the simulated results, we now look at the effect of varying these parasitic elements. In the following simulations, TMAX is fixed at $5 \mathrm{~ns}$. For simplicity, we only display the close-up views in some intervals of a switching cycle.

Fig. 10 shows the simulated results for different values of $L_{r a}$ and $L_{r m}$, and $C_{j o}=83.2 \mathrm{pF}$. The upper panels of Figs. 10 (a) to (c) display erroneous period-2 and period-4 waveforms of $i_{C}$ for relatively small values of inductance with RELTOL $=0.01$. For comparison, we also show in the lower panels the "correct" simulated results with a tighter numerical control, i.e., $\mathrm{RELTOL}=0.001$ and METHOD $=$ Gear. Increasing the parasitic inductance to $150 \mathrm{nH}$, we obtain the "correct" result, as shown in Fig. 10 (d). Thus, we observe that the simulation

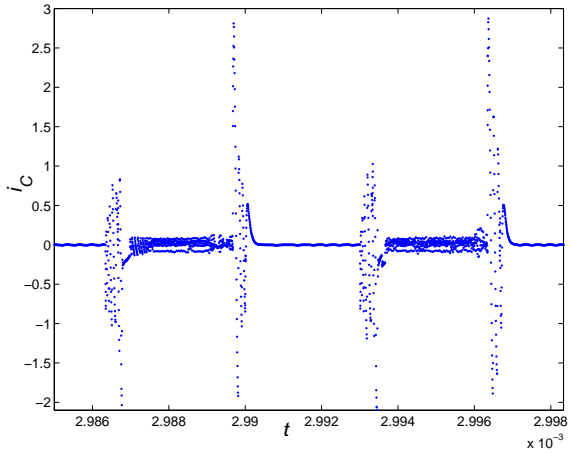

(a)

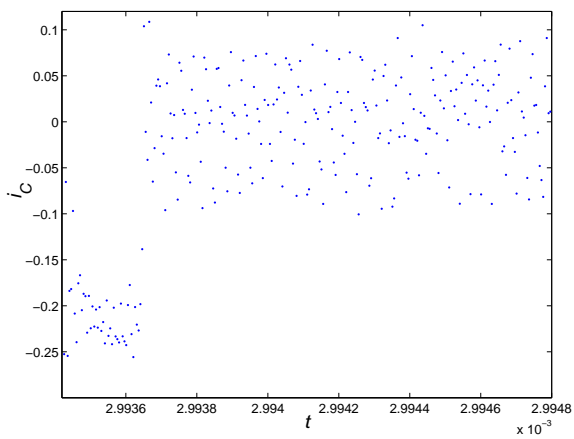

(b)

Fig. 8. (a) Flawed chaotic operation of $i_{C}$ with RELTOL $=0.2$ and (b) close-up view.

results become more "stable" as $L_{r a}$ and $L_{r m}$ are increased.

Fig. 11 shows the simulated results for different values of parasitic capacitance $C_{j o}$, while $L_{r a}$ and $L_{r m}$ are kept at $5 \mathrm{nH}$. The upper panels of Figs. 11 (a) to (c) display erroneous periodic waveforms of $i_{C}$ for relatively small values of $C_{j o}$ with RELTOL $=0.01$. Increasing the capacitance to $400 \mathrm{pF}$, period-2 operation is observed, as shown in Fig. 11 (c). The corresponding "correct" results for different values of $C_{j o}$ are also presented in the lower panels in Figs. 11 (a) to (c) for the purpose of comparison. Further increasing $C_{j o}$ to $2400 \mathrm{pF}$, we obtain the "correct" result, as shown in Fig. 11 (d). Thus, the simulation results again become more "stable" as the parasitic capacitance is increased.

\section{Application: IDENTIFICATION OF Operation BOUNDARIES}

Of engineering importance is the ranges of parameters that correspond to different types of results, flawed and correct. To address this issue, we need to identify the operation boundaries in some appropriate parameter space. We have performed a large number of simulations for different sets of parameters, which enable operation boundaries to be identified. It should be noted that although numerical results are presented here for a particular circuit, the general procedure is applicable to any circuit to yield similar information.

Fig. 12 (a) displays the boundaries of operations in the 2dimensional parameter space of $\log _{10}$ RELTOL versus TMAX. It can be observed that the simulated results become more 


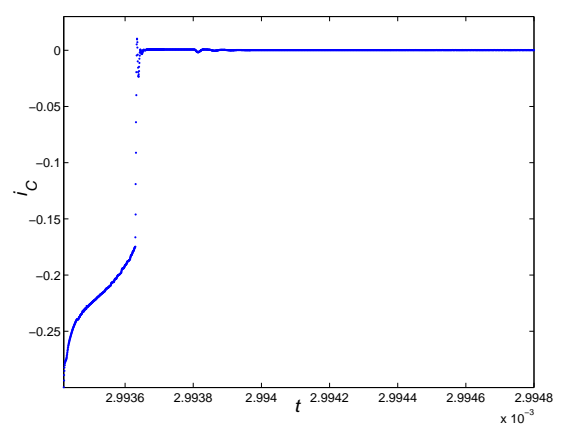

(a)

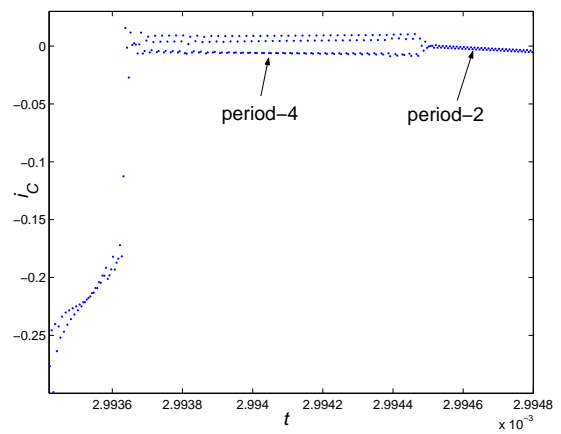

(c)

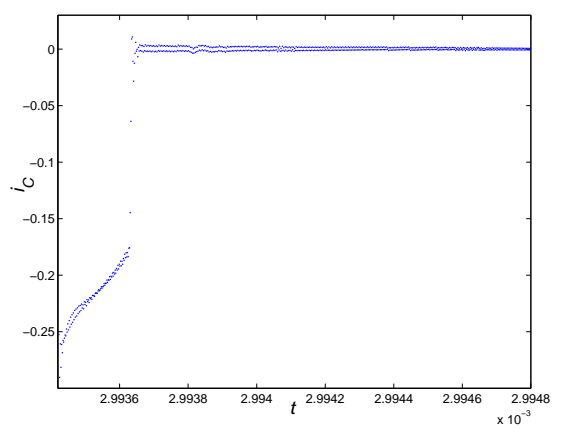

(b)

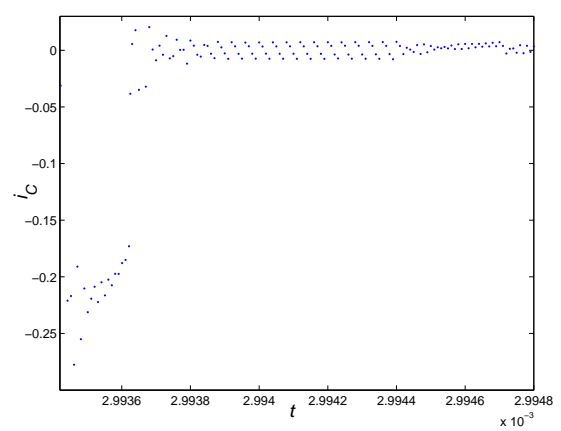

(d)

Fig. 9. (a) "Correct" simulation of $i_{C}$ with TMAX $=0.5 \mathrm{~ns}$; (b) flawed period-2 operation of $i_{C}$ with TMAX $=2$ ns; (c) flawed period-4 operation of $i_{C}$ with TMAX $=5 \mathrm{~ns}$; (d) flawed periodic operation of $i_{C}$ with TMAX $=10 \mathrm{~ns}$.
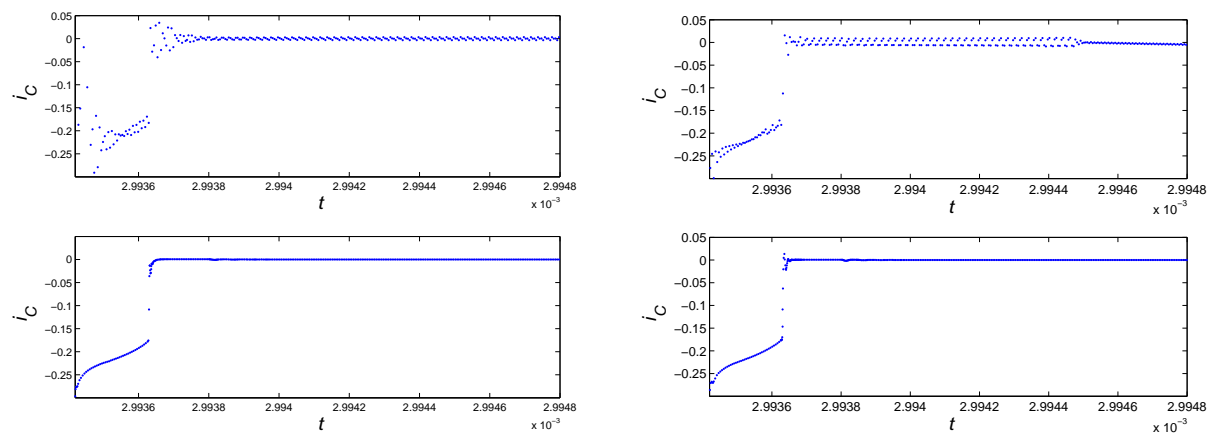

(a)

(b)
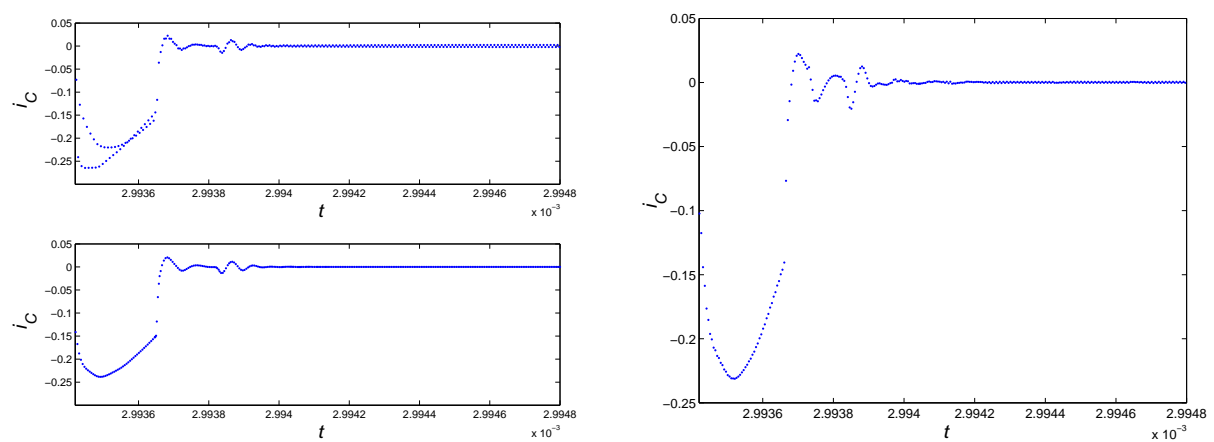

(c)

(d)

Fig. 10. Simulated waveforms of $i_{C}$ with different $L_{r a}$ and $L_{r m}$, and $C_{j o}=83.2 \mathrm{pF}$. (a) $L_{r a}=L_{r m}=1 \mathrm{nH}$. Upper panel: flawed periodic operation with RELTOL $=0.01$; lower panel: "correct" result with RELTOL $=0.001$ and METHOD $=$ Gear. (b) $L_{r a}=L_{r m}=5 \mathrm{nH}$. Upper panel: flawed periodic operation with RELTOL $=0.01$; lower panel: "correct" result with RELTOL $=0.001$ and METHOD $=$ Gear. (c) $L_{r a}=L_{r m}=80 \mathrm{nH}$. Upper panel: flawed periodic operation with RELTOL $=0.01$; lower panel: correct result with RELTOL $=0.001$ and METHOD $=$ Gear. (d) "Correct" simulation with $L_{r a}=L_{r m}$ $=150 \mathrm{nH}$ and $\mathrm{RELTOL}=0.01$. 

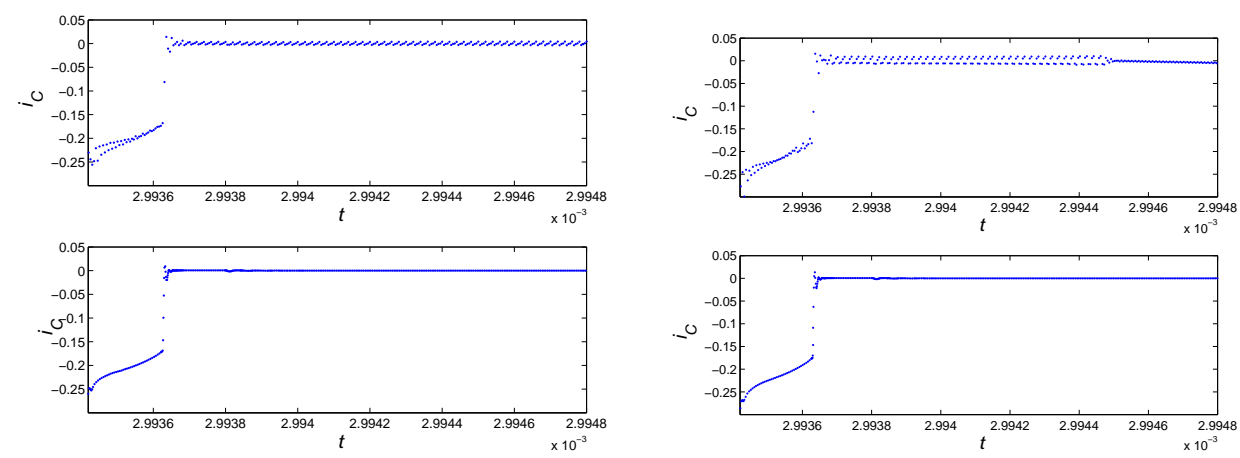

(a)

(b)
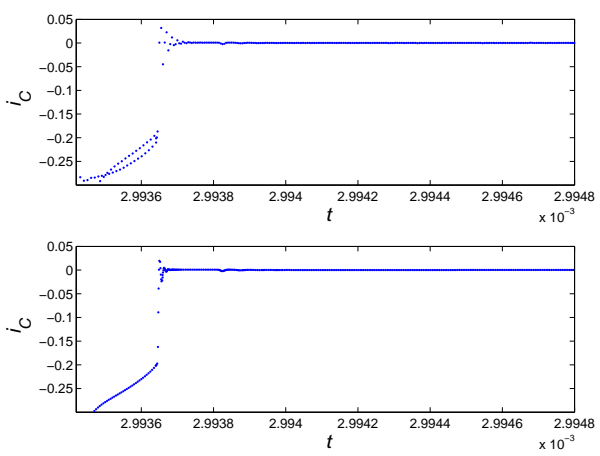

(c)

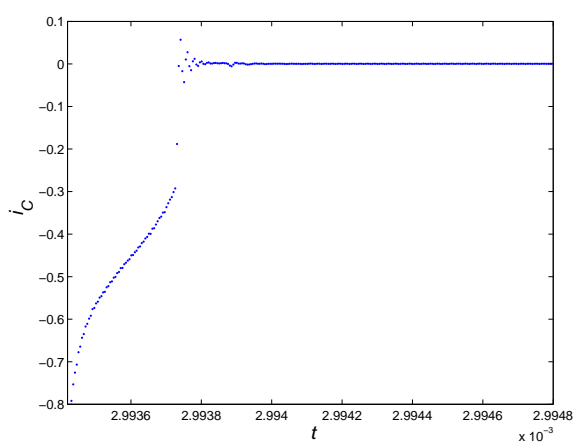

(d)

Fig. 11. Simulated waveforms of $i_{C}$ with different $C_{j o}$, and $L_{r a}=L_{r m}=5 \mathrm{nH}$. (a) $C_{j o}=15 \mathrm{pF}$. Upper panel: flawed periodic operation with RELTOL $=$ 0.01; lower panel: "correct" result with RELTOL $=0.001$ and METHOD $=$ Gear. (b) $C_{j o}=83.2 \mathrm{pF}$. Upper panel: flawed periodic operation with RELTOL $=0.01$; lower panel: "correct" result with RELTOL $=0.001$ and METHOD $=$ Gear. (c) $C_{j o}=400 \mathrm{pF}$. Upper panel: flawed periodic operation with RELTOL $=0.01$; lower panel: "correct" result with RELTOL $=0.001$ and METHOD $=$ Gear. (d) "Correct" simulation with $C_{j o}=2400 \mathrm{pF}$ and RELTOL $=0.01$.

"unstable" as TMAX and RELTOL are increased, which is consistent with the observations made earlier in Sections IIIA and III-B.

Figs. 12 (b) and (c) display the boundaries of operations in the 2-dimensional parameter space of $\log _{10}$ RELTOL versus $L_{r a}, L_{r m}$ and $C_{j o}$. Here, we observe that the simulated results become generally more "stable" as the parasitic inductance and capacitance are increased. The correct-flawed simulation boundaries in the space of parasitic capacitance versus parasitic inductance for different values of RELTOL are shown in Fig. 12 (d), where TMAX is fixed at $5 \mathrm{~ns}$. For a certain value of RELTOL, we find that the numerically obtained boundary curves are generally consistent with the curve $L C=\alpha_{i}$, where $\alpha_{i}$ is a constant. The correct simulated results should then correspond to the region defined by $L C>\alpha_{i}$. Since the parasitic resonant frequency is given by

$$
f=\frac{1}{2 \pi \sqrt{L C}}
$$

the above observation clearly indicates that for given values of RELTOL and TMAX, there is a critical parasitic resonant frequency $f_{c}$ below which the simulated results are correct. Fig. 13 displays the three-dimensional boundary surface in the 3-dimensional space of the parasitic resonant frequency, RELTOL and TMAX. In this figure, correct simulations correspond to the region below the surface and erroneous simulations correspond to system parameters above the surface.

\section{CONCLUSION}

The SPICE simulator, along with the switching converter it imitates, has been studied in terms of the bifurcation behavior of the simulated results. A dynamical system viewpoint is taken to examine the SPICE simulated results for different parameter values. It has been found that SPICE exhibits period-doubling bifurcation and chaos when parameters are not properly chosen, giving erroneous results which do not reflect the true behavior of the circuits being simulated. Our main conclusion is that SPICE is itself a dynamical system which is controlled by a number of parameters whose variations cause the simulated results to manifest various behaviors. We also find that the system's behavior is affected by the parasitic resonant frequency of the circuit. In this paper, we have considered four parameters in particular, namely, relative error tolerance, maximum step size, parasitic inductance and parasitic capacitance. We have performed a thorough investigation which identifies various system parameters that affect the integrity of the simulation results and the parameter boundaries that define a region in which trustworthy results can be guaranteed. The results obtained here can facilitate parameter selection for guaranteeing correct operations. 


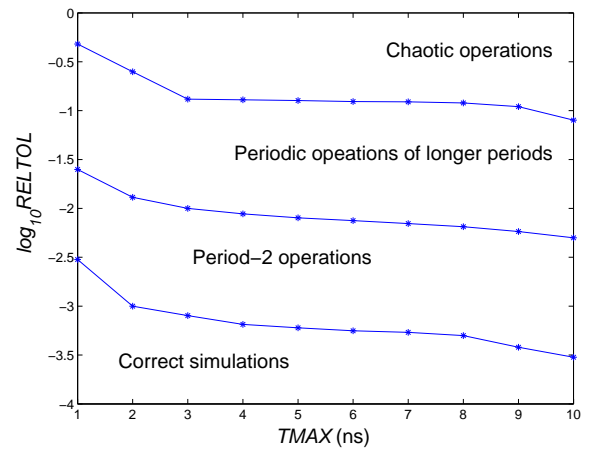

(a)

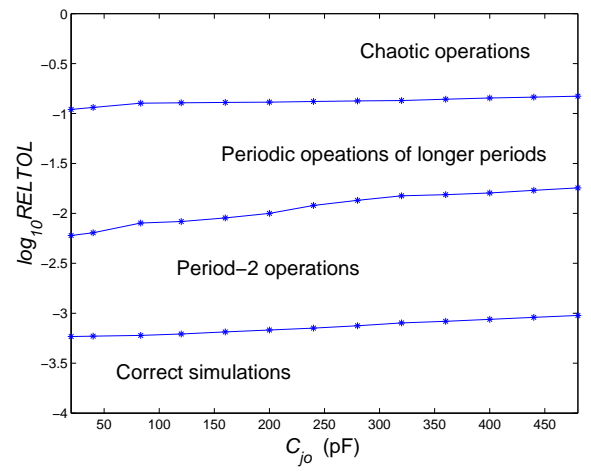

(c)

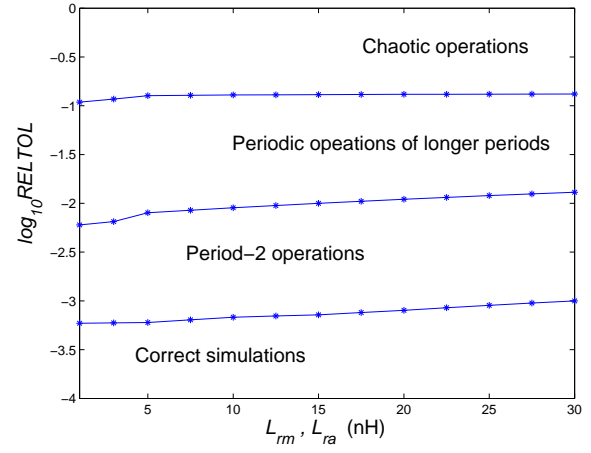

(b)

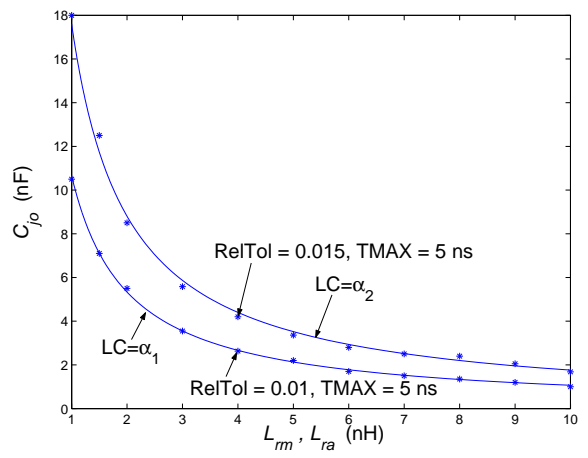

(d)

Fig. 12. Boundaries of operations in 2-dimensional parameter space of (a) $\log _{10}$ RELTOL vs TMAX; (b) $\log _{10}$ RELTOL vs parasitic inductance; (c) $\log _{10}$ RELTOL vs parasitic capacitance; (d) parasitic capacitance vs parasitic inductance for two different values of RELTOL. Simulation of "correct" results corresponds to region above the curves.

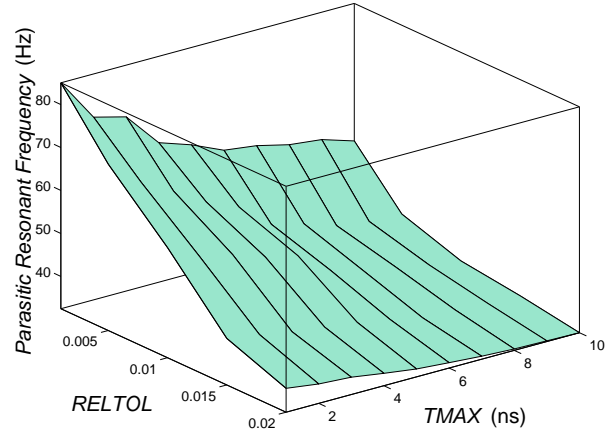

Fig. 13. Correct-flawed simulation boundary surface. "Correct" simulated results correspond to the area below the surface.

\section{ACKNOWLEDGMENTS}

This project is supported by Hong Kong Research Grant Council under a competitive earmarked research grant (No. PolyU 5219/04E).

\section{REFERENCES}

[1] D. Maksimovic, A. M. Stankovic, V. J. Thottuvelil, and G. C. Verghese, "Modeling and simulation of power electronic converters," Proc. IEEE, vol. 89, no. 6, pp. 898-912, June 2001.

[2] T. L. Quarles, SPICE3 Version 3C1 User's Guide, University of California, ERL Memo No. UCB/ERL M89/46, Berkeley, Aprril 1989.
[3] J. Xu, and M. Grotzbach, "Time-domain analysis of half-wave zerocurrent switch quasi-resonant converters by using SPICE," IEEE Trans. Industrial Electron., vol. 40, no. 6, pp. 577-579, December 1993.

[4] A. M. Nassar, A. M. Moselhy, and M. A. El-Gazzar, "Comparison study between a computer aided analysis program using state space technique and SPICE program," Proc. URSI Int. Symp. Signals, Systems, and Electronics, pp. 477-480, October 1995

[5] J. F. Dawson, D. Bozec, D. R. Cullen, L. M. McCormack, and B. W. Flynn, "Simulation of harmonic and conducted interference levels from switched-mode power supplies and phase controllers," Proc. Int. Symp. Electromagnetic Compatibility, 2004.

[6] R. D. Castro, "Solving SPICE problems," IEE Proc. Pt. G, vol. 135 , no. 4, pp 177-178, August 1988.

[7] Z. Q. Shang, "SPICE: Surviving problems in circuit evaluation," IEE Colloquium on the Convergence Problem in SPICE, pp. 10/1-10/5, 30 June 1993

[8] E. Yilmaz and M. M. Green, "Some standard SPICE DC algorithms revisited: why does SPICE still not converge?" Proc. IEEE Int. Symp. Circuits and Systems, pp. 286-289, 1999.

[9] K. G. Nichols, T. J. Kazmierski, M. Zwolinski, and A. D. Brown, "Overview of SPICE-like circuit simulation algorithms," IEE Proc. Circuits, Devices and Systems, vol. 141, no. 4, pp. 242-250, Aug. 1994

[10] C. K. Tse, Complex Behavior of Switching Power Converters, Boca Raton: CRC Press, 2003.

[11] C. K. Tse and M. di Bernardo, "Complex behavior of switching power converters," Proc. IEEE, vol. 90, no. 5, pp. 768-781, May 2002.

[12] K. T. Alligood, T. D. Sauer and J. A. Yorke, Chaos: An Introduction to Dynamical Systems, New York: Springer Verlag, 1996.

[13] Y. H. Leu and C. L. Chen, "Improved asymmetrical half-bridge converter using a tapped output inductor filter," IEE Proc. Electrical Power Applications, vol. 150, no. 4, pp. 417-424, April 2003.

[14] Y. H. Leu, C. L. Chen, and T. M. Chen, "Analysis and design of twotransformer asymmetrical half-bridge converter," IEEE Power Electron. Specialists Conf. Record, pp. 943-948, June 2002. 